\title{
Dynamic structure factors of a Heisenberg model ferrofluid
}

\author{
I.Mryglod ${ }^{1}$, R.Folk ${ }^{2}$, S.Dubyk ${ }^{3}$, Yu.Rudavskii ${ }^{3}$ \\ 1 Institute for Condensed Matter Physics \\ of the National Academy of Sciences of Ukraine, \\ 1 Svientsitskii Str., 290011 Lviv, Ukraine \\ 2 Institut für Theoretische Physik, Universität Linz, \\ Altenbergerstr. 69, A-4040 Linz, Österreich \\ 3 State University "Lvivska Politekhnika", \\ 12 Bandera Str., 290013 Lviv, Ukraine
}

Received July 11, 1998

Using the results of our previous papers [Physica A 220 (1995) 325; Physica A 234 (1996) 129], where the generalized transport equations for a Heisenberg-like model of a ferrofluid were derived and the hydrodynamic collective mode spectrum has been studied, in this work we consider the expressions for hydrodynamic time correlation functions in the limit of small wavenumbers $k$ and frequencies $\omega$. The analytical results for the time correlation functions of 'particle density-particle density' and 'spin densityspin density', being exact in the hydrodynamic limit, are obtained. The expressions for the corresponding dynamical structure factors are analyzed.

Key words: ferrofluid, hydrodynamics, collective modes, time correlation function, dynamic structure factor

PACS: 05.20.-y, 61.10.Dp, 75.50.Mm

\section{Introduction}

Models of a magnetic liquid are of interest in the theory of magnetism because liquid magnets may have ideal soft-magnetic properties due to their isotropy. Starting from the 80th thermodynamic, structural and dynamic properties of magnetic fluids were studied in many papers. The equilibrium behaviour for a Heisenberg model of ferrofluid has been considered in [1,2]. Dynamic properties of liquid ferromagnets were studied mainly within phenomenological theories (see, e.g. [3,4]). However, some of the results obtained were self-contradictory. For example, one can note that the expressions for sound velocity found within two main groups of phenomenological theories, belonging to the so-called 'co-rotational' and 'co-deformational' models of a ferrofluid, differ even qualitatively [4]. Hence, the problem arose to study the 
hydrodynamic behaviour using rigorous statistical treatment.

In [5] with the help of Zubarev's method of nonequilibrium statistical operator we have derived the generalized transport equations, the equations for time correlation functions and collective mode spectrum for a model of magnetic fluids with isotropic interparticle interactions in an inhomogeneous external magnetic field. These equations have been used for the subsequent investigation of hydrodynamic collective modes in [6]. In particular, it was found that the sound velocity of a Heisenberg-like model ferrofluid at a constant magnetic field is isotropic and can be expressed via an adiabatic compressibility at constant magnetization. In addition to the hydrodynamic sound and heat modes known for simple liquids, the spin diffusion mode with purely real eigenvalue has been discovered. The microscopic expressions for the generalized thermodynamic quantities and generalized transport coefficients have been derived as well.

In this paper we report analytical results for the time correlation functions (TCFs) of 'particle density-particle density' and 'spin density-spin density' obtained in the hydrodynamic limit. The Fourier transforms of these functions are of special interest to the theory, because they are the dynamic structure factors which can be measured by scattering experiments.

\section{Theoretical framework}

We consider a Heisenberg model of ferrofluid described by the Hamiltonian

$$
\hat{H}=\sum_{i}^{N} \frac{\mathbf{p}_{i}^{2}}{2 \mathrm{~m}}+\frac{1}{2} \sum_{i \neq j}^{N} V\left(\left|\mathbf{r}_{i j}\right|\right)-\frac{1}{2} \sum_{i \neq j}^{N} J\left(\left|\mathbf{r}_{i j}\right|\right) \mathbf{S}_{i} \mathbf{S}_{j}-h \sum_{i}^{N} S_{i}^{z}
$$

where the first two terms describe a "liquid" subsystem as a simple classical liquid and the other ones contribute from a "magnetic" subsystem with Heisenberg-like interactions $[1,2,5,6]$.

In [6] it has been shown that the matrix equation for Laplace transforms $\tilde{F}(k, z)$ of the equilibrium time correlation functions $F(k, t)$,

$$
F(k, t)=\int_{0}^{1} \mathrm{~d} \tau\left\langle\Delta \hat{Y}(k) \exp \{-\mathrm{i} \hat{L} t\} \rho_{0}^{\tau} \Delta \hat{Y}^{+}(k) \rho_{0}^{-\tau}\right\rangle \equiv\left(\hat{Y}(k), \mathrm{e}^{-\mathrm{i} \hat{L} t} \hat{Y}^{+}(k)\right)
$$

has a form

$$
\left\{z I-\mathrm{i} \Omega_{0}(k)+\tilde{\phi}(k, z)\right\} \tilde{F}(k, z)=F(k),
$$

where $F(k)=F(k, 0), \tilde{\phi}(k, z)$, and i $\Omega_{0}(k)$ are the matrix of static correlation functions, the matrix of memory functions and the frequency matrix, respectively. The set of orthogonal hydrodynamic variables $\hat{Y}(k)=\left\{\hat{n}_{k}, \hat{p}_{k}, \hat{h}_{k}, \hat{s}_{k}\right\}$ for longitudinal fluctuations could be obtained from the microscopic densities of conserved quantities (densities of particles' number $\hat{n}_{k}$, momentum $\hat{p}_{k}$, energy $\hat{e}_{k}$ and magnetic moment $\hat{m}_{k}$ ) by the orthogonalization Gram-Schmidt procedure [6]. For variables 
$\hat{Y}(k)$ the matrix $F(k)$ is diagonal with the elements which in the hydrodynamic limit could be written as follows

$$
\begin{array}{r}
F_{n n}=\left.N S(k)\right|_{k \rightarrow 0} \approx N n T k_{\mathrm{B}} \varkappa_{T, h}, \quad F_{p p}=N \frac{\mathrm{m}}{\beta}=V \rho T k_{\mathrm{B}}, \\
F_{h h}=\left.\frac{1}{k_{\mathrm{B}} \beta^{2}} C_{n, m}(k)\right|_{k \rightarrow 0} \approx N k_{\mathrm{B}} T^{2} c_{n, m}, \quad F_{s s}=\left.\frac{1}{\beta} \chi_{T, n}(k)\right|_{k \rightarrow 0} \approx N k_{\mathrm{B}} T \bar{\chi}_{T, n},
\end{array}
$$

where $\varkappa_{T, h}$ is an isothermal compressibility at constant field $h ; \bar{\chi}_{T, n}$ and $c_{n, m}$ are a susceptibility and a specific heat at constant volume per one particle; and $\rho=\mathrm{m} n=$ $\mathrm{m} N / V$ is a mass density.

The elements of frequency matrix i $\Omega_{0}(k)=\left(\mathrm{i} \hat{L} \hat{Y}(k), \hat{Y}^{+}(k)\right)\left(\hat{Y}(k), \hat{Y}^{+}(k)\right)^{-1}$ in the hydrodynamic limit are also expressed [6] via the thermodynamic quantities, namely

$$
\mathrm{i} \Omega_{0}^{\mathrm{H}}=\mathrm{i} k\left(\begin{array}{cccc}
0 & \frac{1}{\mathrm{~m}} & 0 & 0 \\
\frac{1}{n \varkappa_{T, h}} & 0 & \frac{\beta_{P, m}}{n \varkappa_{T, m} c_{n, m}} & \frac{\pi_{P, T}}{n \varkappa_{T, h} \bar{\chi}_{T, n}} \\
0 & \frac{T \beta_{P, m}}{\rho \varkappa_{T, m}} & 0 & 0 \\
0 & \frac{\pi_{P, T}}{\rho \varkappa_{T, h}} & 0 & 0
\end{array}\right) \text {, }
$$

where $P$ is a pressure and

$$
\beta_{P, m}=\frac{1}{V}\left(\frac{\partial V}{\partial T}\right)_{P, m}, \quad \pi_{P, T}=\frac{1}{V}\left(\frac{\partial V}{\partial h}\right)_{P, T}
$$

are the thermal expansion and magnetostriction coefficients, respectively.

The elements of memory functions matrix are related to the transport coefficients of a Heisenberg magnetic liquid. Taking into account the symmetry properties, one finds in the hydrodynamic limit $\left.\tilde{\phi}(k, z)\right|_{k \rightarrow 0} \approx \tilde{\phi}(k, 0)=\phi_{\mathrm{H}}(k)$, where

$$
\phi_{\mathrm{H}}(k)=k^{2}\left(\begin{array}{cccc}
0 & 0 & 0 & 0 \\
0 & \frac{\eta_{l}}{\rho} & 0 & 0 \\
0 & 0 & \frac{\lambda}{n c_{n, m}} & \frac{L_{s h}}{n \bar{\chi}_{T, n}} \\
0 & 0 & \frac{L_{s h}}{n T c_{n, m}} & \frac{L_{s s}}{n \bar{\chi}_{T, n}}
\end{array}\right) .
$$

The transport coefficients $\eta_{l}, \lambda, L_{s h}=L_{h s}$, and $L_{s s}$ are longitudinal viscosity, thermal conductivity, thermomagnetic diffusion, and spin diffusion, respectively. In [6] the microscopic Green-Kubo-like expressions for these coefficients have been derived. 
The hydrodynamic collective mode spectrum can be found by solving eigenvalue problem for generalized hydrodynamic operator $T_{\mathrm{H}}(k)=\left\|T_{i j}(k)\right\|=-\mathrm{i} \Omega_{0}^{\mathrm{H}}(k)$ $+\phi_{\mathrm{H}}(k)$. By a similar manner the solutions for the Laplace transforms $\tilde{F}(k, z)$ of hydrodynamic TCFs can be written in the form

$$
\tilde{F}_{i j}(k, z)=\sum_{\alpha} \frac{G_{i j}^{\alpha}(k)}{z+z_{\alpha}(k)},
$$

where $G_{i j}^{\alpha}(k)$ are the weight coefficients describing the contribution due to collective excitation $z_{\alpha}(k)$. The coefficients $G_{i j}^{\alpha}(k)$ are simply expressed via the elements of the eigenvectors matrix $\hat{\mathbf{X}}=\left\|\hat{\mathrm{X}}_{i, \alpha}(k)\right\|$, having been found for $T_{\mathrm{H}}(k)$, namely

$$
G_{\alpha}^{i j}=\hat{\mathrm{X}}_{i, \alpha} \hat{\mathrm{X}}_{\alpha, j}^{-1} F_{j j}(k) \text {. }
$$

The matrix $\hat{\mathbf{X}}^{-1}$ is the inverse of $\hat{\mathbf{X}}=\left\|\hat{\mathbf{X}}_{\alpha}\right\|$. Using solution (2.9), one gets for the dynamic structure factor $S(k, \omega)$ of a magnetic fluid the following expression

$$
S(k, \omega)=\frac{1}{\pi} \operatorname{Re} \tilde{F}_{n n}(k, \mathrm{i} \omega)=\frac{1}{\pi} \operatorname{Re} \sum_{\alpha} \frac{G_{n n}^{\alpha}(k)}{\mathrm{i} \omega+z_{\alpha}(k)} .
$$

Similar expression can be also written for the magnetic dynamic structure factor $S_{m}(k, \omega)$, which describes the magnetic moment fluctuations.

\section{Results and discussion}

Eigenvalues of the matrix $T_{\mathrm{H}}(k)$ give a hydrodynamic collective mode spectrum. We have obtained [6]: two complex-conjugate sound modes $z_{s}^{ \pm}(k)= \pm \mathrm{i} k v_{s} k+\Gamma k^{2}$, the hydrodynamic heat mode $z_{h}(k)=D_{h} k^{2}$, and the hydrodynamic spin-diffusion mode $z_{m}(k)=D_{m} k^{2}$, where $v_{s}=\gamma_{m} /\left(\rho \varkappa_{T, m}\right)=(\partial P / \partial \rho)_{S, m}$ is an adiabatic sound velocity at constant magnetization $m$ and $\gamma_{m}=c_{P, m} / c_{n, m}$. The explicit expressions for the damping coefficients $\Gamma, D_{h}, D_{m}$ are given in [6]. To calculate the eigenvectors $\hat{\mathbf{X}}_{\alpha}=\left\|\hat{\mathbf{X}}_{i, \alpha}(k)\right\|$, the matrix perturbation theory with respect to small parameter $k$ was used. Thus, one has all the needed quantities for the subsequent study of the hydrodynamic TCFs.

The hydrodynamic time correlation functions of 'particle density-particle density' and 'spin density-spin density' are of interest in our study, because they can be extracted from scattering experiments. Their Fourier transforms are the dynamic structure factor $S(k, \omega)$ and the magnetic dynamic structure factor $S_{m}(k, \omega)$, respectively. The solutions for these functions can be written as follows

$$
\begin{gathered}
\frac{S(k, \omega)}{S(k)}=\frac{\delta_{T}}{2 \pi \gamma_{m}} \sum_{\alpha=+,-} \frac{\Gamma k^{2}-\alpha k\left(\omega / v_{s}+\alpha k\right) b_{n}}{\left(\omega+\alpha k v_{s}\right)^{2}+\left(\Gamma k^{2}\right)^{2}}+\frac{1}{\pi} \sum_{\alpha=h, m} \frac{\mathcal{G}_{n, \alpha} D_{\alpha} k^{2}}{\omega^{2}+\left(D_{\alpha} k^{2}\right)^{2}} \\
\frac{S_{m}(k, \omega)}{S_{m}(k)}=\frac{1-\delta_{T}}{2 \pi \gamma_{m}} \sum_{\alpha=+,-} \frac{\Gamma k^{2}-\alpha k\left(\omega / v_{s}+\alpha k\right) b_{m}}{\left(\omega+\alpha k v_{s}\right)^{2}+\left(\Gamma k^{2}\right)^{2}}+\frac{1}{\pi} \sum_{\alpha=h, m} \frac{\mathcal{G}_{m, \alpha} D_{\alpha} k^{2}}{\omega^{2}+\left(D_{\alpha} k^{2}\right)^{2}}
\end{gathered}
$$


where $\delta_{T}=\varkappa_{T, m} / \varkappa_{T, h}$ and

$$
\begin{aligned}
& b_{n}=\frac{\eta_{l}}{\rho}-3 D_{s}, \quad b_{m}=b_{n}+2 \frac{L_{s s}}{n \bar{\chi}_{T, n}}+2 \frac{L_{s h} \beta_{P, m}}{\pi_{P, T} \delta_{T} n c_{n, m}}, \\
& \mathcal{G}_{h}^{n}=\frac{\delta_{T}}{\gamma_{m}\left(D_{m}-D_{h}\right)}\left(\frac{\eta_{l}}{\mathrm{~m} n}-2 D_{s}-\left(1-\frac{\gamma_{m}}{\delta_{T}}\right) D_{m}\right), \\
& \mathcal{G}_{m}^{n}=\frac{\delta_{T}}{\gamma_{m}\left(D_{h}-D_{m}\right)}\left(\frac{\eta_{l}}{\mathrm{~m} n}-2 D_{s}-\left(1-\frac{\gamma_{m}}{\delta_{T}}\right) D_{h}\right), \\
& \mathcal{G}_{h}^{m}=\frac{1}{\gamma_{m}\left(D_{m}-D_{h}\right)}\left(\frac{\lambda \delta_{T}}{n c_{n, m}}-\left(\delta_{T}+\gamma_{m}-1\right) D_{h}\right), \\
& \mathcal{G}_{m}^{m}=\frac{1}{\gamma_{m}\left(D_{h}-D_{m}\right)}\left(\frac{\lambda \delta_{T}}{n c_{n, m}}-\left(\delta_{T}+\gamma_{m}-1\right) D_{m}\right) .
\end{aligned}
$$

We end with a few remarks:

(i) the sound velocity of a Heisenberg model ferrofluid is isotropic and can be expressed via an adiabatic compressibility at constant magnetization. In general the spectrum of hydrodynamic collective modes is isotropic and for a Heisenberg model of ferrofluids with isotropic spin-spin interactions does not depend on the mutual orientation of an external magnetic field $h$ and a wavevector $\mathbf{k}$;

(ii) all the thermodynamic quantities as well as the transport coefficients in the expressions given above depend on $h$. For $h=0$ and in the paramagnetic state the expressions for the dynamical structure factors (3.1) and (3.2) have the most simple form. For instance, only the spin-diffusion mode will contribute to $S_{m}(k, \omega)$, and it does not contribute to $S(k, \omega)$, so that $S(k, \omega)$ has formally the same structure as for a simple fluid [7];

(iii) for nonzero values of $h$ the function $S_{m}(k, \omega)$ has additional contributions due to sound modes. As it is seen from (3.2) the weight of these contributions depends on the factor $\left(1-\delta_{T}\right)$, which is proportional to $h^{2}$ for small values $h$. This result can be testified by appropriate scattering experiments;

(iv) the results obtained are directly applicable to the theory of polar liquids with isotropic interactions. The hydrodynamics of polar liquids and ferrofluids becomes formally identical [4] if one notes the correspondence between "electric field" and "magnetic field" and between "polarization" and "magnetization";

(v) the Landau-Placzek ratios [7] of the integrated intensity of the Reyleight central peak to those of the Brillouin side peaks both for $S(k, \omega)$ and $S_{m}(k, \omega)$ can be easily found from the expressions (3.1) and (3.2). Note that the central peak has two separate Lorentian components due to contributions from the head and spindiffusion modes. These results can be used for interpretation the neutron scattering experiments as well.

Acknowledgements. This study is supported in part by the Fonds für Förderung der wissenschaftlichen Forschung under Project P 12422 TPH. 


\title{
References
}

1. Vakarchuk I.A., Ponedilok G.V., Rudavskii Yu.K. Theory of liquid magnets. // Theor. Math. Phys., 1984, vol. 58, p. 291-302 (in Russian).

2. Vakarchuk I.A., Rudavskii Yu.K., Ponedilok G.V. Free energy of the amorphous ferromagnets with Heisenberg exchange interaction and liquid-like disorder. // Phys. Stat. Sol. B, 1985, vol. 128, p. 231-242.

3. Akhiezer I.A., Akhiezer I.T. Oscillations of a ferromagnet liquid. // Sov. Phys. JETP, 1984, vol. 59, No. 1, p. 68-70 (in Russian).

4. Hubbard J.B., Stiles P.J. Hydrodynamics of magnetic and dielectric colloidal systems. // J. Chem. Phys., 1986, vol. 84, No. 12, p. 6955-6968.

5. Mryglod I.M., Tokarchuk M.V., Folk R. On the hydrodynamic theory of a magnetic liquid. I. General description. // Physica A, 1995, vol. 220, p. 325-348.

6. Mryglod I.M., Folk R. On the hydrodynamic theory of a magnetic liquid. II. Hydrodynamic modes in the Heisenberg fluid. // Physica A, 1996, vol. 234, p. 129-150.

7. Boon J.P., Yip S. Molecular Hydrodynamics. New-York, McGraw-Hill Inc., 1980.

\section{Динамічні структурні фактори для гайзенбергівського модельного ферофлюїду}

\author{
І.Мриглод ${ }^{1}$, Р.Фольк ${ }^{2}$, С.Дубик ${ }^{3}$, Ю.Рудавський ${ }^{3}$ \\ 1 Інститут фізики конденсованих систем НАН України, \\ 290011 Львів, вул. Свенціцького, 1 \\ 2 Інститут теоретичної фізики, Університет м.Лінц, \\ Альтенбергштрассе 69, А-4040 Лінц, Австрія \\ 3 Державний університет “Львівська політехніка", \\ 290013 Львів, вул. С.Бандери 12
}

Отримано 11 липня 1998 р.

Використовуючи результати попередніх робіт [Physica A 220 (1995) 325; Physica A 234 (1996) 129], де були отримані узагальнені рівняння переносу та досліджено спектр гідродинамічних колективних збуджень, у цій роботі розглядаються вирази для часових кореляційних функцій в границі малих значень хвильового вектора і частоти. Для часових кореляційних функцій “густина-густина" та "спінова густина-спінова густина" отримані аналітичні результати, які є асимптотично точними в гідродинамічній границі. Аналізуються вирази для відповідних динамічних структурних факторів.

Ключові слова: ферофлюїд, гідродинаміка, колективні моди, часові кореляційні функції, динамічний структурний фактор.

PACS: $05.20 .-y, 61.10 . D p, 75.50 . M m$ 